\title{
A SEARCH FOR NON-THERMAL EMISSION IN THE HII REGIONS OF THE MAGELLANIC CLOUDS
}

\author{
S.W. AMY \\ School of Physics \\ University of Sydney \\ Sydney NSW 2006 \\ Australia
}

ABSTRACT. The $843 \mathrm{MHz}$ radio survey of the Magellanic Clouds with the Molonglo Observatory Synthesis Telescope (MOST) has provided continuum images with a resolution of about $45 \operatorname{arcsec}$ for many HII regions. By comparing these radio data with $\mathrm{H}$-alpha data regions of non-thermal emission may be found within HII regions. 